\author{
(D) Sunit Kumar Gupta, \\ (D) Mussavvir Agha, \\ (D) Ankur Sharma, \\ (D) Varuna Vyas
}

\section{Refractory Hypotension Despite High Dose Inotropic Support: An Unusual Cause}

Received/Gelis Tarihi : 22.02.2019

Accepted/Kabul Tarihi : 17.09.2019

${ }^{(C)}$ Copyright 2019 by Turkish Society of Intensive Care Turkish Journal of Intensive Care published by Galenos Publishing House.
Keywords: Central venous catheter, hypotension, ultrasound
Sunit Kumar Gupta, Mussavvir Agha

Department Of Anesthesiology \& Intensive Care,

Aiims, Jodhpur, India

Ankur Sharma

Department Of Trauma \&

Emergency(anesthesiology), Aiims, Jodhpur, India

Varuna Vyas

Department Of Pediatrics, Aiims, Jodhpur, India

Ankur Sharma (凶),

Department Of Trauma \&

Emergency(anesthesiology), Aiims, Jodhpur, India

E-mail : ankuranaesthesia@gmail.com

Phone : +919654045653

ORCID ID : orcid.org/0000-0001-9339-6988

\section{Dear Editor,}

Central venous cannulation is common invasive procedure that is executed in operating rooms, ICU's and wards. It is usually put in hemodynamically unstable patients or patients undergoing major surgical procedures. It is a secure venous access for infusion of inotropes, vasoactive drugs, electrolytes, fluid, CVP (central venous pressure) monitoring, temporary hemodialysis, pumlonary artery cannulation or when no peripheral access can be secured. This procedure is associated with many complications including malposition (1). Thus it is important to ensure it's proper placement. This is a report that shows a rare malpositioning of venous catheter in internal jugular vein which resulted into a cause of refractory hypotension despite high dose inotropic support attached to it.

This patient was a case of carcinoma ovary, psueudomyxoma with uterine prolapsed and was planned for cytoreductive surgery. Anaesthesia was induced by experienced anesthesiology team with peripheral venous access as per hospital protocol. After intubation, central venous cannulation was done in internal jugular vein (IJV) by ultrasound (USG) guidance, blood flow was checked in all three ports and 7.5 Fr catheter was fixed at $12 \mathrm{~cm}$ by suturing. The duration of surgery was 7 hours and patient had 3 episodes of hypotension, which was managed with fluid bolus through peripheral venous access. After the 
surgery, patient was shifted to ICU (intensive care unit) for further monitoring. In immediate post operative period, patient required inotropic support, but despite giving inotropes, patient's blood pressure was not able to be maintained. Patient's chest Xray was done and central line was found to be turning on to itself and going in upwards direction (Figure 1a). Immediately IJV was recannulated with 7.5 Fr catheter under USG guidance and blood was checked to be coming from all ports. Post procedure Xray was also done immediately to localize it's position, which was found normal(Figure 1b).

Misplaced Central venous cannulation is a common complication faced by anesthesiologist all over the world. Central venous catheter(CVC) have been found to be misplaced in normal or rarely anomalous structures(1-4). The various sites of CVC malpostion and their clinical features are: carotid artery ( hypotension and hemorrhagic shock); azygos vein ( pleural effusion, pulmonary edema, dyspnea, chest pain, back pain, and cardiac tamponade); persistent leftsided superior vena cava (cardiac tamponade); internal mammary vein ( shoulder or arm pain); vertebral vein ( thrombosis, endothelial damage, and fluid leakage); extradural space (severe back pain); pericardium (hemopericardium,

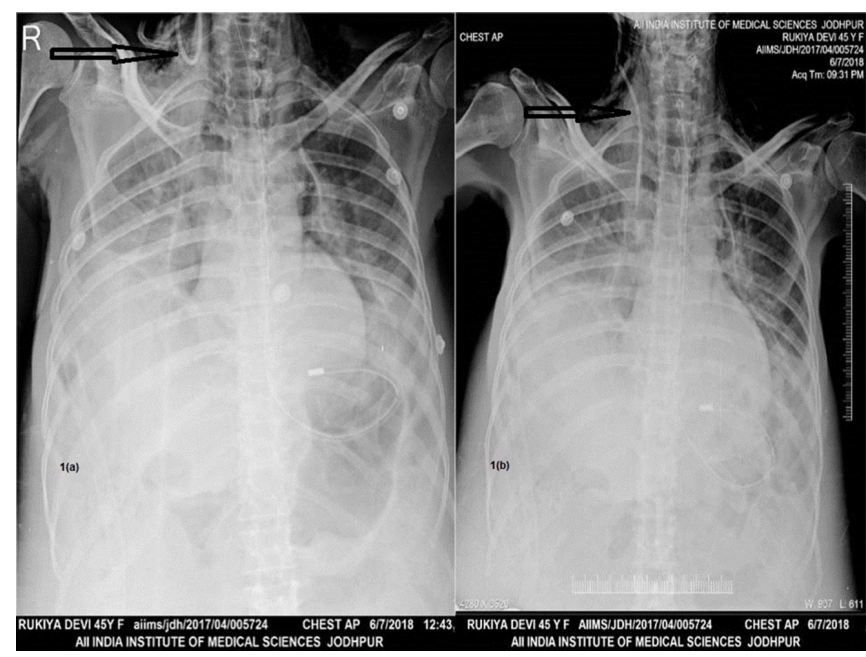

Figure 1a, 1b. Central venous catheter looping back of itself and after repositioning fatal ventricular fibrillation), pleural space (dyspnea, pneumothorax, chest pain, and back pain); mediastinum (Chest pain); thoracic duct(infusion mediastinum and chylothorax) (5). There can be "ear-gurgling" or "water running" sensation and headache if CVC is malpositioned near the intracranial structures(6).

In our case, the angle of the internal jugular vein and subclavian vein, facilitated the catheter looping back of itself by pushing the j-shaped tip of the guidewire upward. This bending of catheter led to it's occlusion, although it was put under USG guidance. This malposition of CVC catheter resulted into a scenario of refractory hypotension despite high dose inotropic support. Kamlipur et al in their observational study on 116 patients comparing USG and chest radiography for detecting the correct location of $\mathrm{CVC}$, found sensitivity of $98 \%$ and specificity of $69 \%$ for USG with positive and negative predictive values were 95\% and $85 \%$, respectively(7). They concluded that despite close concordance between USG and chest radiography, USG is not a suitable alternative for standard chest radiography in detecting CVC location. Further they added that USG can be a useful for detecting CVC location on a real-time basis in the operating room because of it's high sensitivity and acceptable specificity. In a recent meta-analysis(8) of 15 studies, bedside ultrasound was found faster than radiography at identifying pneumothorax after central venous catheter insertion and it reduced mean central venous catheter confirmation time by 58.3 minutes. Chui et al (9) in a recent study on 6,875 patient who underwent USG guided CVC catheter insertion concluded that routine post procedural X-ray is unnecessary.

USG has aided in CVC insertion in many ways. However, fully reliable on USG for localising vein and guiding the needle is not enough and can not prevent malposition of catheter and it can lead to a catastrophic situation. Our message to readers is to scan along the vein to localise the CVC catheter even after it seems apparently normal. We should also not forget the importance of Post CVC insertion chest X-ray to visualize it's tip. 


\section{References}

1. Şahutoğlu C, Pestilci Z, Kocabaş S, Aşkar FZ, Sunal SÖ, Çevik AG. A Venous Catheter Complication: Venous Perforation and Lung Injury. Turk J Anaesthesiol Reanim. 2014;42:270-72.

2. Ozakin E, Can R, Acar N, Baloglu Kaya F, Cevik AA. An Evaluation of Complications in Ultrasound-Guided Central Venous Catheter Insertion in the Emergency Department. Turk J Emerg Med. 2016;14:53-8.

3. Tekelioglu U. Malpositions of central venous catheter: three case reports. Turkish Journal of Thoracic and Cardiovascular Surgery 19:276-8.
4. Akıncı B, Duyu M, Alkılıç L, Yılmaz Karapınar D, Karapınar .Inferior Petrosal Sinus Thrombosis in a Child due to Malposition of Central Venous Catheter: A Case Report. Med Princ Pract. 2017;26:579-81.

5. Wang L, Liu ZS, Wang CA. Malposition of Central Venous Catheter: Presentation and Management. Chin Med J 2016;129:227-34.

6. Gilner LI. The "ear-gurgling" sign. N Engl J Med. 1977;29:1301.

7. Kamalipour H, Ahmadi S, Kamali K, Moaref A, Shafa M, Kamalipour P. Ultrasound for Localization of Central Venous Catheter: A Good Alternative to Chest X-Ray?. Anesth Pain Med. 2016;6:e38834.

8. Ablordeppey EA, Drewry AM, Beyer $A B$, Theodoro DL, Fowler SA, Fuller $\mathrm{BM}$ et al. Diagnostic accuracy of central venous catheter confirmation by bedside ultrasound versus chest radiography in critically ill patients: a systematic review and meta-analysis. Crit Care Med. 2017 ;45:715-24.

9. Chui J, Saeed R, Jakobowski L, Wang W, Eldeyasty B, Zhu F et al. Is Routine Chest X-Ray After Ultrasound-Guided Central Venous Catheter Insertion Choosing Wisely?: A Population-Based Retrospective Study of 6,875 Patients. Chest. 2018; 154:148-56. 